\title{
Endogenous differential information
}

\author{
Sebastián Cea-Echenique $^{1}{ }_{(D)} \cdot$ Carlos Hervés-Beloso $^{2}$. \\ Juan Pablo Torres-Martínez ${ }^{3}$
}

Received: 12 February 2015 / Accepted: 1 October 2015 / Published online: 15 October 2015 (C) Springer-Verlag Berlin Heidelberg 2015

\begin{abstract}
We include endogenous differential information in a model with sequential trade and incomplete financial participation. Agents update information through market signals given by commodity prices and asset deliveries. Information acts over admissible strategies and consumption tastes, allowing discontinuities in preferences and choice sets. Therefore, equilibrium may cease to exist. However, internalizing the compatibility between information and consumption through preferences, and without requiring either financial survival assumptions or fully revealing prices, equilibrium existence can be ensured.
\end{abstract}

\footnotetext{
We would like to thank Jean-Marc Bonnisseau, Bernard Cornet, and João Correia-da-Silva for helpful discussions. We specially thank the detailed comments and suggestions of an anonymous referee.

S. Cea-Echenique acknowledges financial support from Conicyt and University of Chile;

C. Hervés-Beloso acknowledges financial support from Research Grants ECO2012-38860-C02-02

(Ministerio de Economia y Competitividad) and RGEA (Xunta de Galicia and FEDER);

J. P. Torres-Martínez acknowledges financial support from Conicyt through Fondecyt Projects 1120294 and 1150207.
}

$凶 \quad$ Sebastián Cea-Echenique sebastian.cea@psemail.eu

Carlos Hervés-Beloso cherves@uvigo.es

Juan Pablo Torres-Martínez juan.torres@fen.uchile.cl

1 Paris School of Economics - University Paris 1, 106-112 Boulevard de l'Hôpital, 75013 Paris, France

2 RGEA, Facultad de Económicas, Universidad de Vigo, Lagoas Marcosende, 36310 Vigo, Spain

3 Department of Economics, Faculty of Economics and Business, University of Chile, Diagonal Paraguay 257, Santiago, Chile 
Keywords Endogenous differential information $\cdot$ Restricted participation $\cdot$ Rational expectations equilibrium · Incomplete markets

JEL Classification D52 - D53 · D82

\section{Introduction}

There exists a large literature on competitive equilibrium with asymmetric information, a framework introduced by Radner (1968) who extended the classical general equilibrium model by assuming that agents have incomplete and asymmetric information on the future states of nature. In Radner's (1968) model, agents are not able to improve their initial private information, and the model requires information to be consistent with agents' allocations. This is a strong restriction since it is natural to assume that market signals, summarized by future commodity prices, allow agents to update their initial information. Alternatives to this model were studied in further developments, as for instance Radner (1979), who considered beliefs on the future states of nature and showed the existence of rational expectations equilibrium when full-informative prices update agent's initial information (generic equilibrium existence).

Meanwhile, financial imperfections that emerge from regulatory considerations, lack of information or credit risk, induce financial participation constraints. With the aim of studying these situations, the general equilibrium model of incomplete financial markets was extended to scenarios where agents have personalized access to financial opportunities. See, for instance, the pioneering work of Cass (2006) or more recent developments by Cornet and Gopalan (2010), Aouani and Cornet (2009, 2011), and Cornet and Ranjan (2011).

Our aim is to elaborate in this framework, which departs from the model of incomplete markets with numéraire assets and differential information, in two ways: by considering investment constraints, in contrast to the usual credit restrictions, and allowing an endogenous update of agent's private information. On the one hand, when there is restricted participation in financial markets, for instance due to the lack of information, there is no reason to block the access to the missing information that may be available. ${ }^{1}$ Consequently, we will take into account the endogenous information given by market's signals. On the other hand, the fact of incorporating the information given by asset returns avoids inconsistencies between the pattern of financial promises and agents' actions that may arise due to asymmetries on agents' initial information (see Example 6).

As in Dubey et al. (1987), we assume that economic activity takes place sequentially in a frame of time which, for the sake of simplicity, is summarized in two periods. Initially, agents act according to their private information. This produces economic outcomes that could benefit agents with finer information. In this initial period, and due to the lack of information, some group of agents create assets that could avoid some of

\footnotetext{
1 Faias and Moreno-García (2010) analyze the properties of a noninformative equilibrium price in the context of real assets.
} 
the restrictions that appear in some models like Radner (1968). ${ }^{2}$ Indeed, we maintain the scenario of incomplete financial participation. Thus, the asset deliveries update the involved agents' initial information. Additionally, in the first period, individuals are restricted to trade in a subset of the financial market which is compatible with their own-already updated-information and some agents could benefit again from their finer information. Once the assets are present in the market, agents forecast future commodity prices which eventually reveal additional information on the future states of nature. Thus, each agent's final information is the result of the update of her initial private information firstly, by the signals given by the initial economic activity and asset returns, and secondly by market signals given by future spot prices.

Therefore, as in the classical model of rational expectations, Radner (1979), in our model agents' private information can be endogenously improved via market's signals. Thus, this framework reflects how agents demand consumption plans that are compatible with their final information. ${ }^{3}$

However, we argue that the primitives of a real economy could be far from being random. Indeed, due to homogeneity of agent's endowments or tastes across states of nature, randomness can be seriously reduced as a consequence of the lack of agents' initial information and the restricted financial participation. This means that in contrast to Radner (1979), the set of nonfully revealing equilibrium prices could be nonnegligible.

As our model explicitly gives room to nonfully revealing prices, we could have lack of continuity of the choice set correspondence due to the fact that agents eventually learn additional information from partially revealing equilibrium prices (see Remark 1 in Sect. 3). This informational discontinuity was already observed by several authors [see Radner (1967) for instance]. In order to overcome this lack of continuity, ${ }^{4}$ some additional assumptions are needed. For it, when expected utility is considered, we contemplate the situation in which individual's state-dependent preferences may depend on the information that spot commodity prices reveal. Although preferences are exogenously given, contingent consumption can depend on the final information available, which may depend on spot prices. Price-dependent preferences are studied in the work by Pollak (1977) and, more recently, in Correia-da-Silva and Hervés-Beloso (2008) for a model of preferences for lists of bundles. Indeed, even in the classical expected utility case, agents' objective functions depend on their information and consequently are, in general, implicitly price dependent (we stress this point in Sect. 5).

In this scenario, we define an equilibrium with endogenous differential information and restricted financial participation and show equilibrium existence. The classical scenario to analyze rational expectations equilibrium, i.e., expected utility, is included in our model. Thus, in order to apply our existence result to this case, it is sufficient that

\footnotetext{
2 For instance, the example of no-trade caused by the lack of information in Correia-da-Silva and HervésBeloso (2009) does not apply if the obvious assets were to be considered.

3 Correia-da-Silva and Hervés-Beloso (2009) showed that compatibility between consumption and information can be endogenized also in another framework, allowing uncertain delivery of commodities and provided that individuals have prudent expectations on market deliveries.

4 Without financial markets, De Castro et al. (2014) introduce the maximin rational expectations equilibrium (MREE) and provide not a generic proof of existence, as Radner (1979), but a universal one as in our work.
} 
the agent's state-dependent preferences are similar across indistinguishable states with respect to the final information. Nevertheless, our result only requires a more general property in objective functions (see Assumption A). In addition, it is assumed that information revealed by small differences in prices does not make dramatic changes in the agent's behavior. We may strengthen this assumption requiring that only significative differences in prices produce changes on agent's tastes. This argument is consistent with preferences taking tendencies into account. As commodity prices are channels to communicate information, differences in prices could be interpreted as signals of how strong a tendency is realized, i.e., the consumption of a commodity is more exclusive in a state where the price is significantly higher.

The remaining part of the paper is organized as follows: In Sect. 2 we introduce the model, and in Sect. 3 we discuss the possibility of making information endogenously compatible. Our main result and a nonexistence example are stated in Sect. 4. We also provide an example that recaptures equilibrium existence when the continuity assumption is satisfied. In Sect. 5 we focus on the expected utility model. We discuss some additional examples in Sect. 6, and finally we conclude with some remarks. The proof of the main result is contained in an "Appendix."

\section{A financial model with endogenous differential information}

Consider a two period economy without uncertainty in the first period, $t=0$, and where one state of nature of a finite set $S$ is realized in the second period, $t=1$. Thus, let $S^{*}=\{0\} \cup S$ be the set of states of nature in the economy, identifying $s=0$ as the only state of nature in the first period. There is a finite set $L$ of perfect divisible and nonstorable commodities that may be traded at each period in spot markets. We implicitly assume that there are many more states of nature than commodities. ${ }^{5}$

Let $p_{s}=\left(p_{s, l} ; l \in L\right)$ be the vector of commodity prices at state of nature $s \in S^{*}$ and $p=\left(p_{s} ; s \in S^{*}\right)$ the set of commodity prices in the economy. Hereinafter, we fix a bundle $\zeta \in \mathbb{R}_{++}^{L}$ and normalize unitary prices in such form that $p_{s} \cdot \zeta=1, \forall s \in S^{*}$. Let $\mathcal{P}_{\zeta}:=\left\{p \in \mathbb{R}_{+}^{L}: p \cdot \zeta=1\right\}$. Thus, the set of commodity prices will be $\mathcal{P}:=\left\{\left(p_{s} ; s \in S^{*}\right) \in \mathbb{R}_{+}^{L \times S^{*}}: p_{s} \in \mathcal{P}_{\zeta}, \forall s \in S^{*}\right\}$.

There is a finite set $J$ of numéraire assets indexed to the bundle $\zeta{ }^{6}$ Each asset $j \in J$ is issued at the first period, has a unitary price $q_{j}$, and makes promises contingent to the states of nature, $\left(R_{s, j} \zeta ; s \in S\right) \in \mathbb{R}_{+}^{L \times S} \backslash\{0\}$. Let $q:=\left(q_{j} ; j \in J\right) \in \mathbb{R}_{+}^{J}$.

There is a finite set of agents, denoted by $I$. Each individual $i \in I$ may have incomplete information about the realization of the uncertainty, as she only distinguishes states of nature that are in different elements of a partition $\mathbb{P}^{i}$ of $S$, which constitutes her initial private information. Endowments of agent $i$ are given by a

\footnotetext{
5 The requirement of more states of nature than commodities is not relevant in this work since our results do not depend on it. However, Correia-da-Silva and Hervés-Beloso (2014) showed that allowing agents to also trade ex-post in spot markets, generically, the informational asymmetries become irrelevant if there are at least as many commodities as states of nature. See also Correia-da-Silva (2015).

${ }^{6}$ Note that we require the bundle $\zeta$ to have strictly positive coordinates, whereas the general case of numéraire assets allows for nonnegatives coordinates. This normalization is standard in the case of just one commodity in each state.
} 
bundle $w^{i}=\left(w_{s}^{i} ; s \in S^{*}\right) \in \mathbb{R}_{++}^{L \times S^{*}}$ whose restriction to coordinate in $S$ denoted $w_{\mid S}^{i}:=\left(w_{s}^{i} ; s \in S\right)$ is $\mathbb{P}^{i}$-measurable. ${ }^{7}$ The only channel that individuals have to improve their private information is through market signals that are commodity prices and asset deliveries.

Participation in investment clubs or personalized loan scenarios are captured by the fact that individuals may have limited access to financial contracts. Thus, agent $i$ can trade assets in a subset $J^{i} \subseteq J$. We assume that asset returns are signals that may be used to update information before the trade takes place. Thus, for each $i \in I$, the partition $\mathbb{P}^{i}$ incorporates all the information generated by the return of assets in $J^{i}$. That is, for any $j \in J^{i}$, the vector $\left(R_{s, j} ; s \in S\right)$ is $\mathbb{P}^{i}$-measurable. In addition, although the financial participation can be incomplete, any contract $j \in J$ can be traded for at least one agent. That is, $J=\bigcup_{i \in I} J^{i}{ }^{8}$ We also assume that the information required to recognize the realization of the uncertainty can be obtained by the pooling of individuals information, i.e., $\bigvee_{i \in I} \mathbb{P}^{i}=\{\{s\} ; s \in S\}$. $^{9}$

The financial access each agent has is given by nonredundant assets. That is, for each $i \in I$ the family of vectors $\left\{\left(R_{s, j}\right)_{s \in S}: j \in J^{i}\right\}$ is linearly independent. Therefore, we allow redundancy in the market at an aggregate level— e.g., financial intermediationbut there is no redundancy when we consider the individual access to financial markets.

We assume that the new information available could affect individuals' beliefs about the occurrence of states of nature and their tastes about contingent consumption. The process of adapting agent's private information happens at the same time that each agent adapts her individual preference. Given prices $p \in \mathcal{P}$, let $\tau(p)$ be the partition of $S$ generated by commodity prices in the second period $p_{\mid S}:=\left(p_{s} ; s \in S\right)$. Then, the final private information of agent $i$ is given by the partition $\mathbb{P}^{i} \vee \tau(p)$. Thus, individual preferences may depend endogenously on the information transmitted by commodity prices. Therefore, each agent $i \in I$ has a price-dependent utility function $V^{i}: \mathcal{P} \times \mathbb{R}_{+}^{L \times S^{*}} \rightarrow \mathbb{R}$

Each agent $i \in I$ selects her consumption by choosing an informational and budgetary compatible allocation $\left(x_{s}^{i} ; s \in S^{*}\right) \in \mathbb{R}_{+}^{L \times S^{*}}$, implemented through a financial position $\theta^{i}=\left(\theta_{j}^{i} ; j \in J^{i}\right) \in \mathbb{R}^{J^{i}}$. More precisely, given prices $(p, q) \in \mathcal{P} \times \mathbb{R}_{+}^{J}$, the objective of an agent $i \in I$ is to maximize her objective function $V^{i}$ by choosing a consumption vector and a portfolio in her choice set, defined as the collection of vectors $\left(x^{i}, \theta^{i}\right) \in \mathbb{E}^{i}:=\mathbb{R}_{+}^{L \times S^{*}} \times \mathbb{R}^{J^{i}}$ such that $\left(x_{s}^{i} ; s \in S\right)$ is $\mathbb{P}^{i} \vee \tau(p)$-measurable, and

\footnotetext{
7 Given a partition $\mathbb{P}$ of $S$, a vector $\left(v_{s} ; s \in S\right)$ is $\mathbb{P}$-measurable if $v_{s}=v_{s^{\prime}}$ for any pair of states of nature $s$ and $s^{\prime}$ which belongs to the same element of the partition $\mathbb{P}$.

8 Notice that as in Seghir (2011) we do not impose any kind of financial survival assumption. That is, we do not assume that each agent has access to some amount of credit through any asset $j \in J$.

9 Since preferences will endogenize the information compatibility requirement (see Assumption A below), we do not need to assume that for any $s \in S$ there is $i \in I$ that distinguishes it, i.e., $\{s\} \in \mathbb{P}^{i}$. This is a traditional assumption on static general equilibrium models with differential information, and it is used to ensure that (under monotonicity of preferences) the equilibrium price of any contingent commodity contract is strictly positive.
} 


$$
p_{0} x_{0}^{i}+\sum_{j \in J^{i}} q_{j} \theta_{j}^{i} \leq p_{0} w_{0}^{i}, \quad p_{s} x_{s}^{i} \leq p_{s} w_{s}^{i}+\sum_{j \in J^{i}} R_{s, j} \theta_{j}^{i}, \quad \forall s \in S
$$

The collection of $\left(x^{i}, \theta^{i}\right) \in \mathbb{E}^{i}$ satisfying the budget constraints above is denoted by $B^{i}(p, q)$, while the collection of vectors $\left(x^{i}, \theta^{i}\right) \in \mathbb{E}^{i}$ for which $\left(x_{s}^{i} ; s \in S\right)$ is $\mathbb{P}^{i} \vee \tau(p)$-measurable is denoted by $\mathcal{I}^{i}(p)$. Therefore, given $(p, q) \in \mathcal{P} \times \mathbb{R}_{+}^{J}$, the choice set of agent $i \in I$ is $B^{i}(p, q) \cap \mathcal{I}^{i}(p)$.

Definition 1 An equilibrium with endogenous differential information and restricted financial participation is given by prices $(\bar{p}, \bar{q}) \in \mathcal{P} \times \mathbb{R}_{+}^{J}$, allocations and portfolios $\left(\left(\bar{x}^{i}, \bar{\theta}^{i}\right) ; i \in I\right) \in \prod_{i \in I} \mathbb{E}^{i}$ such that

(i) For any agent $i \in I,\left(\bar{x}^{i}, \bar{\theta}^{i}\right) \in \mathbb{E}^{i}$ maximizes the utility function $V^{i}(\bar{p}, \cdot)$ among the allocations in the choice set $B^{i}(\bar{p}, \bar{q}) \cap \mathcal{I}^{i}(\bar{p})$.

(ii) The following market clearing conditions hold,

$$
\sum_{i \in I}\left(\bar{x}_{s}^{i}-w_{s}^{i}\right)=0, \quad \forall s \in S^{*} ; \quad \sum_{i \in I(j)} \bar{\theta}_{j}^{i}=0, \quad \forall j \in J,
$$

where, for any asset $j \in J, I(j):=\left\{i \in I: j \in J^{i}\right\}$.

\section{Endogenously compatible information}

As it was mentioned above, when agents self-restrict their consumption decisions to those that are informational compatible, the presence of endogenous information compromises the continuity of individuals' choice set correspondence and equilibrium may fail to exist. Following, we will illustrate the difficulties that may appear in order to ensure equilibrium existence in a model with endogenous differential information and where agents' consumption plans are restricted by the availability of information.

Remark 1 Note that the choice set correspondence does not have a closed graph. Fix an agent $i \in I$ that is not fully informed (i.e., $\mathbb{P}^{i} \neq\{\{s\} ; s \in S\}$ ), and consider a sequence of commodity prices $\left\{p_{n}\right\}_{n \geq 1} \subset \mathcal{P}$ that converges to $\bar{p}$. Assume that there is a partition $\mathbb{Q}$ strictly finer than $\mathbb{P}^{i} \vee \tau(\bar{p})$ such that $\mathbb{Q}=\bigwedge_{n \geq 1} \mathbb{Q}_{n}$ where $\mathbb{Q}_{n}:=\mathbb{P}^{i} \vee \tau\left(p_{n}\right)$ for any $n \geq 1$.

Let $\theta^{i}=0$ and $x^{i}=\left(w_{0}^{i},\left(\alpha_{s} w_{s}^{i} ; s \in S\right)\right)$, where $\left(\alpha_{s} ; s \in S\right) \in(0,1)^{S}$ is $\mathbb{Q}$ measurable but not $\mathbb{P}^{i}$-measurable. Then, independently of $q \in \mathbb{R}_{+}^{J}$, the plan $\left(x^{i}, \theta^{i}\right)$ belongs to $B^{i}\left(p_{n}, q\right) \cap \mathcal{I}^{i}\left(p_{n}\right)$, for any $n \geq 1$. However, $\left(x^{i}, \theta^{i}\right) \notin B^{i}(\bar{p}, q) \cap \mathcal{I}^{i}(\bar{p})$, since this plan is only $\mathbb{Q}$-measurable.

Observe that we only assume that agent $i$ is not fully informed. In that case, there are prices $\bar{p}$ such that $\mathbb{P}^{i} \vee \tau(\bar{p}) \neq\{\{s\} ; s \in S\}$ and sequences of full-informative commodity prices $\left\{p_{n}\right\}_{n \geq 1} \subset \mathcal{P}$ that converges to $\bar{p}$.

Nevertheless, a regularity condition on preferences that ensures individuals' optimal decisions to be compatible with the final information allows to recover the closed graph property of choice sets, avoiding the informational compatibility restriction. 
Given $(i, p) \in I \times \mathcal{P}$, let $\mathcal{S}^{i}(p)=\{a: S \rightarrow S: a$ is bijective and $a(s) \in$ $\left.A_{s}^{i}(p), \forall s \in S\right\}$, where $A_{s}^{i}(p)$ is the element of $\mathbb{P}^{i} \vee \tau(p)$ that contains the state of nature $s$. Next, we elaborate on agent $i$ 's behavior when there is no full information. Note that if the states of nature $s$ and $s^{\prime}$ are in $A_{s}^{i}(p)$, then by definition $p_{s}=p_{s^{\prime}}$, and prices do not give new information to agent $i$ in order to update her preferences. Individuals take optimal decisions regarding all the information available about the realization of the uncertainty. Let $p \in \mathcal{P}$ be the price system and $\mathbb{P}^{i}(s)$ the initial information of agent $i \in I$ with respect to state $s$. The information revealed by prices $p$ is uninformative with respect to the event $\mathbb{P}^{i}(s)$, if and only if $\mathbb{P}^{i}(s)=A_{s}^{i}(p)$, that is $p_{s}=p_{s^{\prime}}$ for all $s^{\prime} \in \mathbb{P}^{i}(s)$. In this situation, agent $i$ does not possess any information to distinguish among states in $\mathbb{P}^{i}(s)$. With more generality, agent $i$ is not fully informed when $A_{s}^{i}(p) \neq\{s\}$, that is, she is unable to distinguish different states in $A_{s}^{i}(p)$.

The following assumption states that agent $i \in I$, being unable to distinguish among states $s$ and $s^{\prime}$, is indifferent between consuming $x_{s}$ in state $s$ and $x_{s}^{\prime}$ in state $s^{\prime}$ or consuming $x_{s}$ in state $s^{\prime}$ and $x_{s}^{\prime}$ in state $s$. Moreover, the assumption ensures that even if an agent may demand any kind of consumption plan in her budget set, at the optimum, her choice will be measurable with respect to her final information. Notice that this assumption is irrelevant when prices are fully informative.

Assumption A For any agent $i \in I$, the objective function $V^{i}: \mathcal{P} \times \mathbb{R}_{+}^{L \times S^{*}} \rightarrow \mathbb{R}$ satisfies: $\forall p \in \mathcal{P}, \forall\left(x_{s}^{i}\right)_{s \in S^{*}} \in \mathbb{R}_{+}^{L \times S^{*}}, \forall a \in \mathcal{S}^{i}(p)$,

$$
V^{i}\left(p, x_{0}^{i},\left(x_{s}^{i}\right)_{s \in S}\right)=V^{i}\left(p, x_{0}^{i},\left(x_{a(s)}^{i}\right)_{s \in S}\right) .
$$

The argument behind Assumption A states that it is not possible for agent $i$ to value differently the same consumption bundle in indistinguishable states. The next result formalizes our arguments.

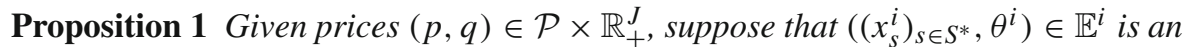
optimal choice for agent $i \in I$ in her budget set $B^{i}(p, q)$. If Assumption A holds, and $V^{i}$ is strictly quasi-concave on consumption, then $\left(x_{s}^{i}\right)_{s \in S}$ belongs to $\mathcal{I}^{i}(p)$.

Proof Suppose that states of nature $s$ and $s^{\prime}$ are in the same element of $\mathbb{P}^{i} \vee \tau(p)$ and that $x_{s}^{i} \neq x_{s^{\prime}}^{i}$. Since $p \in \mathcal{P}$, we have that $p_{s}=p_{s^{\prime}}$. Moreover, as $\mathbb{P}^{i}$ contains the information revealed by the payments of assets in $J^{i}, p_{s} w_{s}^{i}+\sum_{j \in J^{i}} R_{s, j} \theta_{j}^{i}=$ $p_{s^{\prime}} w_{s^{\prime}}^{i}+\sum_{j \in J^{i}} R_{s^{\prime}, j} \theta_{j}^{i}$. Fix $\lambda \in(0,1)$. It follows that $\lambda x_{s}^{i}+(1-\lambda) x_{s^{\prime}}^{i}$ is budget feasible at both states of nature, $s$ and $s^{\prime}$.

Define $z^{i}:=\left(z_{k}^{i}\right)_{k \in S} \in \mathbb{R}_{+}^{L \times S}$ by $z_{k}^{i}=x_{k}^{i}$ when $k \notin\left\{s, s^{\prime}\right\}, z_{s}^{i}=z_{s^{\prime}}^{i}=\lambda x_{s}^{i}+(1-$ ג) $x_{s^{\prime}}^{i}$. In addition, consider the bijection $\tilde{a}: S \rightarrow S$ such that $\tilde{a}(s)=s^{\prime}, \tilde{a}\left(s^{\prime}\right)=s$, and $\tilde{a}(k)=k$ for any $k \notin\left\{s, s^{\prime}\right\}$. Since $V^{i}\left(p, x_{0}^{i},\left(x_{k}^{i}\right)_{k \in S}\right)=V^{i}\left(p, x_{0}^{i},\left(x_{\tilde{a}(k)}^{i}\right)_{k \in S}\right)$, the strict quasi-concavity of $V^{i}$ on consumption ensures that $V^{i}\left(p, x_{0}^{i},\left(z_{k}^{i}\right)_{k \in S}\right)>$ $V^{i}\left(p, x_{0}^{i},\left(x_{k}^{i}\right)_{k \in S}\right)$. Therefore, agent $i$ can improve her utility level choosing the bundle $z^{i}$. A contradiction. 
The following examples provide configurations where Assumption A is satisfied.

Example 1 Suppose for $i \in I$ that there is a state-dependent utility function $u_{s}^{i}$ : $\mathcal{P} \times \mathbb{R}_{+}^{L} \times \mathbb{R}_{+}^{L} \rightarrow \mathbb{R}$ for each $s \in S$ and $v^{i}: \mathcal{P} \times 2^{S} \rightarrow[0,1]$. Denote the set of all monotone set-valued functions on $S$ by $\nabla$, and for a finite $\nabla^{\prime} \subset \nabla$, define

$$
V^{i}(p, x)=\min _{v^{i}(p, \cdot) \in \nabla^{\prime}} \sum_{s \in S} v^{i}(p, s) \cdot u_{s}^{i}\left(p, x_{0}, x_{s}\right) .
$$

In this configuration at given prices $p \in \mathcal{P}$, Assumption $\mathrm{A}$ is fulfilled if the weights $v\left(p, s^{\prime}\right)$ and state-dependent utility functions coincide across indistinguishable states $s^{\prime} \in A_{s}^{i}(p) .{ }^{10}$ Note that expected utility is a particular case of this setting when $\nabla^{\prime}$ contains only one element being a probability measure on $S$.

Example 2 Observe that Assumption A holds for the Cobb-Douglas objective function. For instance, for $i \in I$, let

(a) $V^{i}(p, x)=\sum_{s \in S} \sum_{l \in L} \frac{\alpha_{l}\left(p_{s}\right)}{\#(L \cup S)} \log \left(x_{0, l} x_{s, l}\right)$, where $\alpha_{l}: \mathcal{P}_{\zeta} \rightarrow(0,1)$ for each $l \in L$.

(b) $V^{i}(p, x)=\sum_{s \in S} \frac{\alpha_{s}}{\# S} \log \left(\sum_{l \in L} x_{0, l}+x_{s, l}\right)$, where $\left(\alpha_{s} ; s \in S\right) \in(0,1)^{S}$.

We highlight that Assumption $\mathrm{A}$ is weaker than requiring a condition of measurability in state-dependent preferences. For it, in Example 2(a) if we define $u_{s}\left(p, x_{0}, x_{s}\right)=$ $\sum_{l \in L} \frac{\alpha_{l}\left(p_{s}\right)}{\# L} \log \left(x_{0, l} x_{s, l}\right)$, then we can write $V^{i}(p, x)$ as an expected utility form. Precisely, assuming that states are equiprobable, then $V^{i}(p, x)=\frac{1}{\# S} \sum_{s \in S} u_{S}\left(p, x_{0}, x_{s}\right)$. Thus, in this case state-dependent utility functions are measurable with respect to the final information. On the other hand, state-dependent utility functions may not be measurable with respect to final information and still satisfy Assumption A. For instance, in Example 2(b) if $\left(\alpha_{s} ; s \in S\right)$ is not measurable with respect to $\mathbb{P}^{i} \vee \tau(p)$ if the prevailing prices are $p$.

\section{Equilibrium existence}

In order to state equilibrium existence, we concentrate in a model that also satisfies the hypotheses $\mathrm{B}$ and $\mathrm{C}$ that are described below.

When price dependence is considered in the utility function, continuity in prices is a standard assumption (see footnote 7). Nevertheless, changes on the information revealed by prices may induce discontinuities on the objective function $V^{i}$. To illustrate this possibility, next example sets an economy that fulfills Assumption A, and however, equilibrium fails to exist since there is an agent for whom improving her information matters.

\footnotetext{
10 State-dependent utility functions are measurable with respect to private information if the vector $\left.\left(u_{s}(\cdot, \cdot, \cdot)\right) ; s \in S\right)$ is $\mathbb{P}^{i}$-measurable. Thus, this measurability condition gives no room to preference update if for given $p \in \mathcal{P}$ the partition $\mathbb{P}^{i} \vee \tau(p)$ is finer than $\mathbb{P}^{i}$. In Einy et al. $(2000,2001)$ it is assumed that state-dependent utilities are equal across states that are indistinguishable with respect to the initial information. Thus, Assumption A is fulfilled for every price. Furthermore, note that Assumption A does not impose an expected utility framework.
} 
Example 3 (Nonexistence of Equilibria) Consider an economy with two periods and two states of nature at the second period $\left\{s, s^{\prime}\right\}$. There are two agents $\{A, B\}$ and two commodities $\{x, y\}$. Let $\left(w_{s}^{A}, w_{s^{\prime}}^{A}\right)=((3,1),(1,1))$ and $\left(w_{s}^{B}, w_{s^{\prime}}^{B}\right)=((1,1),(1,1))$ be the initial endowments. Without loss of generality, we pay particular attention to commodity prices $\left(p_{s}, p_{s^{\prime}}\right) \gg 0$ satisfying $p_{s, x}+p_{s, y}=1$ and $p_{s^{\prime}, x}+p_{s^{\prime}, y}=1$ (i.e., we assume that $\zeta=(1,1)$ ).

Agent $A$ initially recognizes the state of nature realized at $t=1$ and maximizes the following function $0.5 x_{s} y_{s}+0.5 x_{s^{\prime}} y_{s^{\prime}}$.

Agent $B$ does not have information to recognize the state of nature at the second period, i.e., $\mathbb{P}^{B}=\left\{\left\{s, s^{\prime}\right\}\right\}$. She maximizes the function

$$
\frac{1}{2} \min \left\{x_{s}, y_{s}\right\}+\frac{1}{2} \min \left\{x_{s^{\prime}}, y_{s^{\prime}}\right\},
$$

whenever prices satisfy $p_{s}=p_{s^{\prime}}$, otherwise she maximizes

$$
\frac{1}{2} x_{s}+\frac{1}{2} x_{s^{\prime}} y_{s^{\prime}}
$$

Since state-dependent utility functions of agent B are equal when prices are noninformative, Assumption A is satisfied.

By monotonicity of preferences, if there are equilibrium prices, then they are strictly positive. Furthermore, for $\left(p_{s}, p_{s^{\prime}}\right) \gg 0$ and with abuse of notation taking $p_{s}=$ $p_{s, x} / p_{s, y},{ }^{11}$ the demands for commodities at prices satisfy

$$
\begin{aligned}
& {\left[\left(x_{s}^{A}, y_{s}^{A}\right) ;\left(x_{s^{\prime}}^{A}, y_{s^{\prime}}^{A}\right)\right]=\left[\left(\frac{3 p_{s}+1}{2 p_{s}}, \frac{3 p_{s}+1}{2}\right) ;\left(\frac{p_{s^{\prime}}+1}{2 p_{s^{\prime}}}, \frac{p_{s^{\prime}}+1}{2}\right)\right] .} \\
& {\left[\left(x_{s}^{B}, y_{s}^{B}\right) ;\left(x_{s^{\prime}}^{B}, y_{s^{\prime}}^{B}\right)\right]= \begin{cases}{[(1,1) ;(1,1)],} & p_{s}=p_{s^{\prime}} \\
{\left[\left(\frac{\left(p_{s}, 1\right) \cdot w_{s}^{B}}{p_{s}}, 0\right) ;\left(\frac{\left(p_{s^{\prime}}, 1\right) w_{s^{\prime}}^{B}}{2 p_{s^{\prime}}}, \frac{\left(p_{s^{\prime}}, 1\right) \cdot w_{s^{\prime}}^{B}}{2}\right)\right],} & p_{s} \neq p_{s^{\prime}} .\end{cases} }
\end{aligned}
$$

If there is an equilibrium in this economy, then we have:

(i) Equilibrium prices satisfy $\bar{p}_{s} \neq \bar{p}_{s^{\prime}}$.

Summing up demands of commodity $x$ in state $s$ and equaling it to the aggregate endowment, we get that $p_{s}=1$. Replicating the procedure for state $s^{\prime}$, we arrive to the conclusion that $p_{s^{\prime}}=1$. A contradiction with the fact that prices are different.

(ii) Equilibrium prices satisfy $\bar{p}_{s}=\bar{p}_{s^{\prime}}$. From market clearing conditions, we get $\bar{p}_{s}=\frac{1}{3}$ as well as $\bar{p}_{s^{\prime}}=1$. A contradiction with the fact that $\bar{p}_{s}=\bar{p}_{s^{\prime}}$.

Thus, in order to obtain equilibrium existence, we assume continuity, local nonsatiation, strict monotonicity, and strict quasi-concavity that are standard assumptions in the literature.

Assumption B For any agent $i \in I$, the utility function $V^{i}$ is continuous on prices and consumption, locally nonsatiated at each state $s \in S^{*}$ and strictly quasi-concave

11 Since demands are homogeneous in prices, this abuse of notation is without loss of generality. 
on consumption. Furthermore, for each $(l, s) \in L \times S^{*}$, there is some $i \in I$ such that $V^{i}$ is strictly increasing in $x_{s, l} \cdot{ }^{12}$

Next, we give a particular example of the functional form of a state-dependent utility function satisfying our assumptions. Particularly, we develop a generalized form of the classic CES utility function such that the elasticity of substitution is not more constant, but depends on prices. Here, we capture equilibrium existence for preferences given in Example 3 if the uninformed agent only cares about price differences that are significatively large. Note that the continuity required in Assumption B is not satisfied in Example 3.

Example 4 (Price-dependent Elasticity of Substitution) Take the configuration of the Example 3. Define a state-dependent utility with a CES form. ${ }^{13}$ That is, for each $k \in\left\{s, s^{\prime}\right\}$, let $\alpha_{k}: \mathcal{P} \rightarrow[0,1]$ be continuous and

$$
u_{k}\left(p, x_{k}, y_{k}\right)=\left[\alpha_{k}(p) x_{k}^{r(p)}+\left(1-\alpha_{k}(p)\right) y_{k}^{r(p)}\right]^{\frac{1}{r(p)}}
$$

where for $0 \leq \epsilon<\delta$, we have $r(p)=\ln \left(\frac{\left\|p_{s}-p_{s^{\prime}}\right\|-\epsilon}{\delta-\epsilon}\right)$ if $\epsilon<\left\|p_{s}-p_{s^{\prime}}\right\|<\delta$, $r(p)=-\infty$ if $\left\|p_{s}-p_{s^{\prime}}\right\| \leq \epsilon$, and $r(p)=0$ if $\left\|p_{s}-p_{s^{\prime}}\right\| \geq \delta$. Replace the objective function of agent $B$ by the continuous function:

$$
V(p, x)=\left\{\begin{array}{ll}
\frac{1}{2} \sum_{k \in\left\{s, s^{\prime}\right\}} u_{k}\left(p, x_{k}, y_{k}\right) & \text { if } \epsilon<\left\|p_{s}-p_{s^{\prime}}\right\|<\delta \\
\frac{1}{2} \lim _{\left\|p_{s}-p_{s^{\prime}}\right\| \rightarrow \epsilon} \sum_{k \in\left\{s, s^{\prime}\right\}} u_{k}\left(p, x_{k}, y_{k}\right) & \text { if }\left\|p_{s}-p_{s^{\prime}}\right\| \leq \epsilon \\
\frac{1}{2} \lim _{\left\|p_{s}-p_{s^{\prime}}\right\| \rightarrow \delta} \sum_{k \in\left\{s, s^{\prime}\right\}} u_{k}\left(p, x_{k}, y_{k}\right) & \text { if }\left\|p_{s}-p_{s^{\prime}}\right\| \geq \delta
\end{array} .\right.
$$

Therefore, when $\left\|p_{s}-p_{s^{\prime}}\right\| \leq \epsilon$, then $u_{k}\left(p, x_{k}, y_{k}\right)$ becomes the function $\min \left\{x_{k}, y_{k}\right\}$, and if $\left\|p_{s}-p_{s^{\prime}}\right\| \geq \delta$, then the state-dependent utility takes a Cobb-Douglas form $x_{k}^{\alpha_{k}(p)} y_{k}^{1-\alpha_{k}(p)}$. We obtain the same objective function given in Example 3 in the case prices are noninformative. Otherwise, we recover the objective function of Agent $B$ when differences in prices are sufficiently large, i.e., $\left\|p_{s}-p_{s^{\prime}}\right\| \geq \delta$ and $\left(\alpha_{s}(p), \alpha_{s^{\prime}}(p)\right)=(1,0.5)$.

When $\epsilon<\left\|p_{s}-p_{s^{\prime}}\right\|<\delta$, demand functions are given by:

$$
x_{s}^{B}(p)=p_{s}^{\frac{1}{r(p)}}\left(p_{s}+1\right)\left[\left(\frac{\alpha_{s}(p)}{1-\alpha_{s}(p)}\right)^{\frac{1}{r(p)-1}}+p_{s}^{\frac{r(p)}{r(p)-1}}\right]^{-1}, \quad s \in\left\{s, s^{\prime}\right\}
$$

\footnotetext{
12 The local nonsatiation property at state $s \in S^{*}$ requires, for each $\left(x_{k}^{i}\right)_{k \in S^{*}} \in \mathbb{R}_{+}^{L \times S^{*}}$ and $\epsilon>0$ the existence of a bundle $y_{s}^{i} \in \mathbb{R}_{+}^{L}$ with $\left\|y_{s}^{i}-x_{s}^{i}\right\|<\epsilon$ and $V^{i}\left(p,\left(x_{k}^{i}\right)_{k \neq s}, y_{s}^{i}\right)>V^{i}\left(p,\left(x^{i}\right)_{k \in S^{*}}\right)$ for every $p \in \mathcal{P}$.

13 The function $g$ is a CES function, if there exist $\gamma \in(0,1)$ and $\rho \in(-\infty, 0) \cup(0,1)$ such that $g(a, b)=\left(\gamma a^{\rho}+(1-\gamma) b^{\rho}\right)^{\frac{1}{\rho}}$.
} 
Therefore, equilibrium equations are:

$$
\frac{3 p_{s}+1}{2 p_{s}}+x_{s}^{B}(p)=4 ; \quad \frac{p_{s^{\prime}}+1}{2 p_{s^{\prime}}}+x_{s^{\prime}}^{B}(p)=2 .
$$

Which equilibrium is $\left(\bar{p}_{s}, \bar{p}_{s^{\prime}}\right) \approx(1,1.5)$ and demands are:

$$
\begin{aligned}
& \left(\left(x_{s}^{A}(\bar{p}), x_{s^{\prime}}^{A}(\bar{p})\right) ;\left(y_{s}^{A}(\bar{p}), y_{s^{\prime}}^{A}(\bar{p})\right)\right)=((2,0.8) ;(2,1.25)) \text { and } \\
& \left(\left(x_{s}^{B}(\bar{p}), x_{s^{\prime}}^{B}(\bar{p})\right) ;\left(y_{s}^{B}(\bar{p}), y_{s^{\prime}}^{B}(\bar{p})\right)\right) \approx((2,1.2) ;(0,0.75) .
\end{aligned}
$$

Incomplete participation in financial markets makes difficult to normalize prices and to ensure that budget set correspondences have a nonempty interior, at the same time. For these reasons, and in order to find endogenous upper bounds on asset prices, it is enough to impose Assumption C on the agents' behavior, which states that a reduction in future consumption (at $t=1$ ) can be compensated by rising the consumption in the first period (at $t=0$ ).

Assumption $\mathbf{C}$ For any agent $i \in I$, given $\sigma \in(0,1)$ there is a continuous mapping $r_{\sigma}: \mathcal{P} \times \mathbb{R}_{+}^{L \times S^{*}} \rightarrow \mathbb{R}_{+}^{L}$, that satisfies: $\forall p \in \mathcal{P}, \forall\left(x_{s} ; s \in S^{*}\right) \in \mathbb{R}_{++}^{L \times S^{*}}$,

$$
V^{i}\left(p, x_{0}+r_{\sigma}(p, x),\left(\sigma x_{s} ; s \in S\right)\right)>V^{i}\left(p,\left(x_{s} ; s \in S^{*}\right)\right) .
$$

Remark 2 Observe that the utility functions in Examples 2 and 4 fulfill Assumptions A, $\mathrm{B}$, and C. Also, the utility function in Example 1 satisfies Assumptions A-C if the state-dependent utilities fulfill it. Assumption $\mathrm{C}$ is satisfied for a great variety of utility functions. It is already satisfied by a monotone utility functions that is unbounded in some commodity consumed in the first period (any Cobb-Douglas utility function or the expected utility with state-dependent Cobb-Douglas preferences).

In Seghir (2011), Assumption C is used to find upper bounds for asset prices; however and in contrast to our model, in that paper they have restrictions on credit opportunities only. Therefore, given restrictions on investment as well as on credit opportunities, we find upper bounds on asset prices using this Assumption (see Lemma 4 in the "Appendix").

Theorem 1 Under Assumptions A, B and C, there exists an equilibrium for the economy with endogenous differential information and incomplete financial participation.

Proof See the "Appendix."

\section{The expected utility}

A particular configuration that is considered in our formulation for the objective function is the expected utility. In order to illustrate our framework in this particular scenario, we assume that all states are equiprobable. Each agent $i \in I$ has a utility function, $\tilde{u}_{s}^{i}: \mathbb{R}_{+}^{L} \times \mathbb{R}_{+}^{L} \rightarrow \mathbb{R}$ representing her preferences in each state $s \in S$. Moreover, if the financial structure is not considered, then our choice set, $B^{i}(p, q) \cap \mathcal{I}^{i}(p)$, 
incorporates the measurability condition and it has the standard configuration of rational expectations equilibrium (REE).

Let $p \in \mathcal{P}$ be the prevailing price system. If $p$ is fully informative, i.e., $A_{s}^{i}(p)=\{s\}$ for all $s \in S$, classical REE assumes agent $i$ receives a utility $\tilde{u}_{s}^{i}\left(x_{0}, x_{s}\right)$ when the state of nature $s$ is reached and the expected utility of agent $i$ is given by ${ }^{14}$ :

$$
V^{i}\left(p, x^{i}\right)=\sum_{s \in S} \frac{1}{\# S} \cdot \tilde{u}_{S}^{i}\left(x_{0}, x_{s}\right) .
$$

Alternatively, i.e., when $A_{s}^{i}(p) \neq\{s\}$, agent $i$ evaluates her utility in state $s$ as a weighted average of the utilities across the states that are indistinguishable of $s$,

$$
u_{[s]}^{i}\left(p, x_{0}, x_{s}\right):=\sum_{s^{\prime} \in A_{s}^{i}(p)} \frac{1}{\# A_{s}^{i}(p)} \cdot \tilde{u}_{s^{\prime}}^{i}\left(x_{0}, x_{s}\right),
$$

and computes her expected utility as follows:

$$
V^{i}\left(p, x^{i}\right)=\sum_{s \in S} \frac{1}{\# S} \cdot u_{[s]}^{i}\left(p, x_{0}, x_{S}\right) .
$$

Thus, even when prices do not affect state-dependent preferences, prices are present in each agent's objective function as a consequence of the update of information. This is because the contribution of the state $s$ to the function $V^{i}$ is $\tilde{u}_{s}^{i}$, if prices are full informative regarding the state of nature or $u_{[s]}^{i}$ otherwise.

In order to accomplish Assumption A, we require additional hypothesis on statedependent utilities that are price (informational) dependent. Einy et al. (2000, 2001) assume that state-dependent utilities (that do not depend on prices) are measurable with respect to initial information. That is, for every price $p \in \mathcal{P}$ and state $s^{\prime} \in \mathbb{P}^{i}(s)$ : $u_{[s]}^{i}(p, \cdot, \cdot):=\tilde{u}_{s^{\prime}}^{i}(\cdot, \cdot)$. This assumption that implies Assumption A and the continuity of the objective function is restrictive since it precludes the possibility of utility update when information matters. Indeed, note that in this configuration the weighted average does not depend on prices.

With the aim to recover the possibility of utility update and ensuring the continuity of the objective function, we will assume that (contrary to the Example 3 where it is a discontinuous function of prices) state-dependent utility functions $u_{s}^{i}, s \in S$, satisfy Assumption B. Furthermore, our result requires expected utility fulfilling Assumption A. For it, a sufficient requirement is that if $p \in \mathcal{P}$ is the prevailing price and $s^{\prime} \in A_{s}^{i}(p)$, then $u_{s}^{i}\left(p, x_{0}, x_{s}\right)=u_{s^{\prime}}^{i}\left(p, x_{0}, x_{s}\right)$, that is, final information measurability. Nevertheless, as in Example 2(b) for state-dependent utilities that are not measurable with respect to final information, Assumption A is still satisfied.

Thus, when prices across states initially indistinguishable for agent $i \in I$ add new information, the state-dependent utility function is updated in a smooth way if Assumption B is required. The assumption prevents the transition via the information

$\overline{14}$ More generally, it is possible to consider beliefs about the realization of the uncertainty (see Example 2). 
revealed by prices from being abrupt, as in Example 3. That is, the smaller the difference in prices between initially indistinguishable states is, the closer the state utility should be to the utility of the other indistinguished states [see Examples 2(a) and 4].

We may strengthen the argument assuming that there is an $\epsilon>0$ such that $u_{s}^{i}\left(p, x_{0}, \cdot\right)$ and $u_{s^{\prime}}^{i}\left(p, x_{0}, \cdot\right)$ for $s^{\prime} \in \mathbb{P}^{i}(s)$ are equal when $\max _{s^{\prime} \in \mathbb{P}^{i}(s)}\left\|p_{s}-p_{s^{\prime}}\right\| \leq \epsilon$ (Example 4 illustrates this possibility). This case represents an scenario where $\epsilon$ differences in prices do not affect at all the state utility. In other words, an $\epsilon>0$ can be understood as the personalized threshold from which the agent subjectively differentiates her preferences across indistinguishable states. Observe that if $\epsilon$ is big enough, we fall in the case where the state utility functions are not price dependent, as in Einy et al. (2000, 2001).

\section{Other examples}

The following examples offer some insights about price informativeness and information compatibility captured in the model. First, we elaborate on nonfully revealing prices. Second, we illustrate the relation between the initial information and the information revealed by the asset that are accessible.

Example 5 (Noninformative equilibrium) Consider an economy with two commodities and utility functions given by

$$
U^{i}\left(\left(x_{s} ; s \in S^{*}\right)\right)=\sum_{s \in S}\left(x_{0,1}^{\beta} x_{0,2}^{1-\beta}+x_{s, 1}^{\beta} x_{s, 2}^{1-\beta}\right), \quad \forall i \in I,
$$

where $\beta \in(0,1)$ is the same for all agents. Then, Assumptions $\mathrm{A}, \mathrm{B}$, and $\mathrm{C}$ hold (see Remark 2). Also, first-order conditions of consumer's $i$ problem at state $s \in S$ would imply that at any equilibrium price $\bar{p}_{s}$,

$$
\frac{\bar{p}_{s, 1}}{\bar{p}_{s, 2}}=\frac{\beta}{1-\beta} \frac{W_{s, 2}}{W_{s, 1}},
$$

where $W_{s, l}=\sum_{i \in I} w_{s, l}^{i}$. Suppose that there is an uninformed agent $i_{0} \in I$ (i.e., $\left.\mathbb{P}^{i_{0}}=\{S\}\right)$. Then, equilibrium prices are noninformative if, and only if, the relative degree of commodity scarcity is constant at the second period, $\frac{W_{s, 2}}{W_{s, 1}}=\frac{W_{s^{\prime}, 2}}{W_{s^{\prime}, 1}}, \forall\left(s, s^{\prime}\right) \in$ $S \times S$, which is a restrictive hypothesis and it is also associated with the absence of aggregated uncertainty. ${ }^{15}$ Thus, for any economy in which this condition does not hold, any equilibrium price will reveal information (at least for the uninformed agent $\left.i_{0}\right)$.

The following proposition illustrates examples of our model in which the equilibrium prices are, at least, partially informative. That is, at given prices $p \in \mathcal{P}$, the partition $\mathbb{P}^{i} \vee \tau(p)$ is finer than $\mathbb{P}^{i}$. For robust partially revealing examples including ambiguity, see Condie and Ganguli (2011).

15 We thank an anonymous referee for pointing out this concept. 
Proposition 2 Under Assumptions A to C, assume that there are two commodities $l$ and $l^{\prime}$ such that the preferences of any agent $i \in I$ can be represented by a utility function

$$
V^{i}(p, x)=\sum_{s \in S} \pi_{s}^{i} v^{i}\left(p, x_{0}^{i},\left(x_{s, k}^{i}\right)_{k \notin\left\{l, l^{\prime}\right\}}, g\left(x_{s, l}^{i}, x_{s, l^{\prime}}^{i}\right)\right)
$$

where $v^{i}$ is differentiable and $g: \mathbb{R}_{+} \times \mathbb{R}_{+} \rightarrow \mathbb{R}$ is a CES function. In addition, suppose that the relative scarcity of commodities $l$ and $l^{\prime}$ differs across states of nature $\left(s, s^{\prime}\right) \in S \times S$ (there is aggregate uncertainty), i.e.,

$$
\frac{\sum_{i \in I} w_{s, l}^{i}}{\sum_{i \in I} w_{s^{\prime}, l}^{i}} \neq \frac{\sum_{i \in I} w_{s, l^{\prime}}^{i}}{\sum_{i \in I} w_{s^{\prime}, l^{\prime}}^{i}}
$$

Then, in any equilibrium commodity prices are at least partially informative.

Proof By Contradiction, assume that equilibrium prices are noninformative. Therefore, for states of nature $s$ and $s^{\prime}$, we have $\bar{p}_{s}=\bar{p}_{s^{\prime}}$. Since $g$ is a CES function, there exist $\gamma \in(0,1)$ and $\rho \in(-\infty, 0) \cup(0,1)$ such that $g(a, b)=\left(\gamma a^{\rho}+(1-\gamma) b^{\rho}\right)^{\frac{1}{\rho}}$. The marginal rate of substitution between the consumption of commodities $l$ and $l^{\prime}$ at node $k \in\left\{s, s^{\prime}\right\}$ is given by $\frac{\gamma\left(\bar{x}_{k, l}^{i}\right)^{\rho-1}}{(1-\gamma)\left(\bar{x}_{k, l^{\prime}}^{i}\right)^{\rho-1}}$. Thus, Karush-Kuhn-Tucker conditions of agent $i$ 's individual problem guarantee that

$$
\bar{x}_{k, l}^{i}=\left(\frac{(1-\gamma)}{\gamma} \frac{\bar{p}_{k, l}}{\bar{p}_{k, l^{\prime}}}\right)^{\frac{1}{\rho-1}} \bar{x}_{k, l^{\prime}}^{i}
$$

Adding across agents, we obtain that

$$
\sum_{i \in I} w_{k, l}^{i}=\left(\frac{(1-\gamma)}{\gamma} \frac{\bar{p}_{k, l}}{\bar{p}_{k, l^{\prime}}}\right)^{\frac{1}{\rho-1}} \sum_{i \in I} w_{k, l^{\prime}}^{i}, \quad k \in\left\{s, s^{\prime}\right\} .
$$

Therefore, as $\bar{p}_{s}=\bar{p}_{s^{\prime}}$, the relative scarcity of commodities $l$ and $l^{\prime}$ coincides at nodes $s$ and $s^{\prime}$, which contradicts our initial assumption.

Example 6 In this example, we will illustrate the importance of the compatibility between the initial information and the information revealed by the asset that an agent can trade. For the sake of simplicity, consider an economy with only one commodity, three states of nature at $t=1$, denoted by $\{u, m, d\}$, and two agents who only receive utility for consumption in the second period. Also, they do not have any initial endowment at $t=0 .{ }^{16}$ Thus, utility functions and endowments are given by

\footnotetext{
16 In order to formally cover configurations without commodity-trading markets in the first period, it is sufficient to include a state dependency in the set of commodities $L$. More precisely, suppose that there is a set of commodities $L_{S}$ for each state $s \in S^{*}$. Under this configuration, Example 6 requires $L_{0}=\emptyset$ inducing no essential changes in our results.
} 


$$
\begin{aligned}
U^{1}\left(x_{u}, x_{m}, x_{d}\right) & =\sqrt{2} \sqrt{x_{u}}+\sqrt{x_{m}}+\sqrt{x_{d}}, \quad\left(w_{u}^{1}, w_{m}^{1}, w_{d}^{1}\right)=(3,3,3) \\
U^{2}\left(x_{u}, x_{m}, x_{d}\right) & =\sqrt{x_{u}}+\sqrt{x_{m}}+\sqrt{2} \sqrt{x_{d}}, \quad\left(w_{u}^{2}, w_{m}^{2}, w_{d}^{2}\right)=(3,3,3) .
\end{aligned}
$$

There are two Arrow securities in the economy. One of them has a unitary price $q_{1}$ and promises to deliver one unit of the commodity at state of nature $s=u$. The other makes a contingent payment of one unit of the commodity at $s=d$ and is negotiated for a unitary price $q_{2}$.

If there is a complete financial participation, the first period budget constraint of agent $i \in\{1,2\}$ is given by $q_{1} z_{1}^{i}+q_{2} z_{2}^{i}=0$, where $z_{j}^{i}$ denotes the position of agent $i$ on asset $j \in\{1,2\}$.

Assume that unitary prices are given by $\bar{q}_{1}=\bar{q}_{2}=1$. Then, the allocations

$$
\left(z_{1}^{1}, z_{2}^{1}, x_{u}^{1}, x_{m}^{1}, x_{d}^{1}\right)=(1,-1,4,3,2), \quad\left(z_{1}^{2}, z_{2}^{2}, x_{u}^{2}, x_{m}^{2}, x_{d}^{2}\right)=(-1,1,2,3,4) .
$$

constitute an equilibrium for the economy.

We argue that if agents are not fully informed-that is, they do not internalize the information revealed by asset payments - the implementation of this equilibrium allocation may not be credible. For instance, assume that $\mathbb{P}^{1}=\{\{u\},\{m, d\}\}$ and $\mathbb{P}^{2}=\{\{u, m\},\{d\}\}$. In order to pay her debt, it is required that agent $i=1$ observes that the state of nature $s=d$ was realized. This would be impossible to accomplish as commodity prices do not communicate information, and there is no other financial signal which allows recognition between states $m$ and $d$. Analogously, to pay her debt, it is required that agent $i=2$ observes some signal that allows her to distinguish between $u$ and $m$, which is an impossible task to accomplish given the financial structure.

\section{Concluding remarks}

In this paper, we elaborate on a model of competitive market with differential information, where agents have restricted participation in incomplete financial markets. Agents, sequentially, are able to add new information from the traded assets, buy commodities in spot markets, and receive the signals given by spot prices allowing them to improve their previous private information.

Our model allows agents to obtain information through the variability of payments in financial markets. Thus, individuals obtain all the information revealed by the awareness conveyed by securities that they can trade. However, there is an incomplete access to financial instruments available in the economy. In order to be consistent with the information transmitted by asset returns and the restrictions that imply the incomplete access to investment opportunities, we ensure that equilibrium exists without the requirement of any kind of financial survival restriction.

We contemplate a scenario where the agents' final information about the realization of uncertainty needs not to be fully revealing. In our model, the final information may have real effects over the agent's capability to implement heterogeneous preferences across states of nature. Equilibrium existence is obtained without imposing any additional compatibility requirement between consumption and information. The 
measurability of optimal bundles is a consequence of the informational-dependent nature of individuals objective functions, since there are no gains for consumption heterogeneity in states of nature that are indistinguishable.

\section{Appendix: Proof of Theorem 1}

To prove equilibrium existence, we first define a generalized game in which agents maximize utility functions in truncated budget sets. Auctioneers choose prices in order to maximize the value of the excess of demand in commodity and financial markets. We prove that this generalized game has a Cournot-Nash equilibrium and also that when the upper bounds on allocations are high enough, any equilibrium of the generalized game will be an equilibrium of our economy.

The generalized game $\mathcal{G}(Q, X, \Theta)$. Given any vector $(Q, X, \Theta) \in \mathbb{R}_{++}^{3}$, we define a game characterized by the following set of players and strategies.

Set of players. There is a finite set of players constituted by

(i) The set of agents of the economy, $I$.

(ii) An auctioneer, $h(s)$, for each $s \in S^{*}$.

We denote the set of players by $H=I \cup H\left(S^{*}\right)$ where $H\left(S^{*}\right):=\left\{h(s): s \in S^{*}\right\}$.

Sets of strategies. Given $\bar{W}:=\max _{(s, l) \in S^{*} \times L} \sum_{i \in I} w_{s, l}^{i}$, define for any $i \in I$,

$$
K^{i}(X, \Theta)=[0, X]^{L} \times[0,2 \bar{W}]^{S \times L} \times[-\Theta, \Theta]^{J^{i}},
$$

and recall $\mathcal{P}_{\zeta}=\left\{p \in \mathbb{R}_{+}^{L}: p \cdot \zeta=1\right\}$. The set of strategies for the players in the generalized game, $\left(\bar{\Gamma}^{h} ; h \in H\right)$, are given by,

(i) For each $h \in I, \bar{\Gamma}^{h}=K^{h}(X, \Theta)$.

(ii) For $h=h(0), \bar{\Gamma}^{h}=\mathcal{P}_{\zeta} \times[0, Q]^{\# J}$

(iii) For $h=h(s)$, with $s \in S, \bar{\Gamma}^{h}=\mathcal{P}_{\zeta}$.

For simplicity, let $\eta^{h}=\left(x^{h}, \theta^{h}\right) \in \bar{\Gamma}^{h}$ be a generic vector of strategies for a player $h \in I ;\left(p_{0}, q\right)$ will denote a generic strategy for the player $h(0)$; and $p_{s}$ a generic strategy for a player $h(s)$, with $s \in S$. Finally, let $\bar{\Gamma}=\prod_{h \in H} \bar{\Gamma}^{h}$ be the space of strategies of $\mathcal{G}(Q, X, \Theta)$. A generic element of $\bar{\Gamma}$ is denoted by $(p, q, \eta)$, where $\eta:=\left(\eta^{h} ; h \in I\right)$ is a generic element of $\prod_{i \in I} \bar{\Gamma}^{i}$.

Admissible strategies. Strategies effectively chosen for players depend on the actions taken by other players, through a correspondence of admissible strategies $\phi^{h}: \bar{\Gamma}_{-h} \rightarrow$ $\bar{\Gamma}^{h}$, where $\bar{\Gamma}_{-h}=\prod_{h^{\prime} \neq h} \bar{\Gamma}^{h^{\prime}}$. Let $(p, q, \eta)_{-h}$ be a generic element of $\bar{\Gamma}_{-h}$. We suppose that

(i) If $h \in I, \phi^{h}\left[(p, q, \eta)_{-h}\right]=B^{h}(p, q) \cap \bar{\Gamma}^{h}$.

(ii) If $h \in H\left(S^{*}\right), \phi^{h}\left[(p, q, \eta)_{-h}\right]=\bar{\Gamma}^{h}$. 
Objective functions. Each player is also characterized by an objective function $F^{h}$ : $\bar{\Gamma}^{h} \times \bar{\Gamma}_{-h} \rightarrow \mathbb{R}_{+}$. We assume that

(i) When $h \in I$ and $\eta^{h}=\left(x^{h}, \theta^{h}\right) \in \bar{\Gamma}^{h}$, then $F^{h}\left(\eta^{h} ;(p, q, \eta)_{-h}\right)=V^{i}\left(p, x^{h}\right)$.

(ii) If $h=h(0)$ and $\left(p_{0}, q\right) \in \bar{\Gamma}^{h}$, then

$$
F^{h}\left(\left(p_{0}, q\right) ;(p, q, \eta)_{-h}\right):=p_{0} \sum_{i \in I}\left(x_{0}^{i}-w_{0}^{i}\right)+\sum_{i \in I} \sum_{j \in J^{i}} q_{j} \theta_{j}^{i} .
$$

(iii) If $h(s) \in H\left(S^{*}\right) \backslash\{h(0)\}$ and $p_{s} \in \bar{\Gamma}^{h}$, then $F^{h}\left(p_{s} ;(p, q, \eta)_{-h}\right):=$ $p_{s} \sum_{i \in I}\left(x_{s}^{i}-w_{s}^{i}\right)$.

We define the correspondence of optimal strategies for each $h \in H, \Psi^{h}: \bar{\Gamma}_{-h} \rightarrow$ $\bar{\Gamma}^{h}$ as

$$
\Psi^{h}\left((p, q, \eta)_{-h}\right):=\underset{y \in \phi^{h}\left((p, q, \eta)_{-h}\right)}{\arg \max } F^{h}\left(y ;(p, q, \eta)_{-h}\right) .
$$

Finally, let $\Psi: \bar{\Gamma} \rightarrow \bar{\Gamma}$ be the correspondence of optimal game response, which is given by $\Psi(p, q, \eta)=\prod_{h \in H} \Psi^{h}\left((p, q, \eta)_{-h}\right)$.

Definition 2 A Cournot-Nash equilibrium for the generalized game $\mathcal{G}(Q, X, \Theta)$ is given by a strategy profile $(\bar{p}, \bar{q}, \bar{\eta}) \in \bar{\Gamma}$ such that $(\bar{p}, \bar{q}, \bar{\eta}) \in \Psi(\bar{p}, \bar{q}, \bar{\eta})$.

In order to prove the existence of equilibrium in the generalized game, we need some properties of the admissible strategy correspondence which the following lemma provides.

Lemma 1 For any $h \in H, \phi^{h}$ is continuous and has nonempty, compact, and convex values.

Proof For each player $h \in H\left(S^{*}\right)$, the correspondence of admissible strategies is constant and, therefore, it is continuous and nonempty. Also, by definition, its values are compact and convex.

On the other hand, for each player $h \in I$, it follows from the definition of the budget set that the correspondence of admissible strategies $\phi^{h}$ has nonempty, compact, and convex values. Since the graph of this correspondence is closed, we obtain upper hemicontinuity. To assure the lower hemicontinuity of $\phi^{h}$, we consider the correspondence $\dot{\phi}^{h}\left((p, q, \eta)_{-h}\right):=i n t_{K^{h}(X, \Theta)} B^{h}(p, q)$, which associates with a vector of commodity and asset prices the set of allocations in $K^{h}(X, \Theta)$ that satisfy all the budget restrictions of agent $h$ as strict inequalities. Note that this correspondence has nonempty values and open graph. Therefore, it is lower hemicontinuous. We know that the closure of $\dot{\phi}^{h}\left((p, q, \eta)_{-h}\right)$, which is equal to $\phi^{h}\left((p, q, \eta)_{-h}\right)$, is also lower hemicontinuous. Therefore, correspondences of admissible strategies $\left(\phi^{h} ; h \in I\right)$ are continuous.

Lemma 2 Under $(A)$ and $(B)$, the set of Cournot-Nash equilibria of $\mathcal{G}(Q, X, \Theta)$ is nonempty. 
Proof By Assumption (A) and (B), each objective function in the game is continuous in all variables and quasi-concave in its own strategy. Also, the sets of strategies are nonempty, compact, and convex. By Lemma 1, admissible correspondence is continuous with nonempty, convex, and compact values. Thus, we can apply Berge's maximum theorem to assure that for each player $h \in H$, the correspondence of optimal strategies, $\Psi^{h}$, is upper hemicontinuous with nonempty, convex, and compact values. Therefore, the correspondence $\Psi$ has closed graph with nonempty, compact, and convex values. Applying Kakutani's fixed-point theorem to $\Psi$, we conclude the proof.

We will prove that for vectors $(Q, X, \Theta) \in \mathbb{R}_{++}^{3}$ for which coordinates are high enough, any equilibrium of the generalized game is an equilibrium for our economy. However, we need to previously find endogenous upper bounds for equilibrium variables.

Lemma 3 For each $(i, s) \in I \times S$, fix a vector $\left(p_{s}, w_{s}^{i}, x_{s}^{i}\right) \in \mathcal{P}_{\zeta} \times \mathbb{R}_{+}^{L} \times \mathbb{R}_{+}^{L}$, with $x_{s}^{i}<\bar{W}$. Then, there exists $A>0$ such that any vector $\left(\kappa_{j}^{i} ; j \in J^{i}\right) \in \mathbb{R}^{J^{i}}$ satisfying

$$
p_{s} x_{s}^{i}=p_{s} w_{s}^{i}+\sum_{j \in J^{i}} R_{s, j} \kappa_{j}^{i}, \quad \forall(i, s) \in I \times S
$$

belongs to $[-A, A]^{\# J^{i}}$. Also, A only depends on $\left(\left(\bar{W}, w_{s}, R_{s, j}\right) ;(s, j) \in S \times J\right)$.

Proof Fix $i \in I$. Note that as $S$ (respectively, $J$ ) is a finite set, by abusing of the notation and identifying it with $\{1, \ldots, S\}$ (respectively, $\left\{1, \ldots, J^{i}\right\}$ ) we can rewrite the conditions in the statement of the lemma in a matricial form:

$$
\left[\begin{array}{c}
p_{1}\left(x_{1}^{i}-w_{1}^{i}\right) \\
\vdots \\
p_{S}\left(x_{S}^{i}-w_{S}^{i}\right)
\end{array}\right]=\left[\begin{array}{ccc}
R_{1,1} & \cdots & R_{1, J^{i}} \\
\vdots & \ddots & \vdots \\
R_{S, 1} & \cdots & R_{S, J^{i}}
\end{array}\right]\left[\begin{array}{c}
\kappa_{1}^{i} \\
\vdots \\
\kappa_{J^{i}}^{i}
\end{array}\right]
$$

Since for $i \in I$ there are no redundant assets in $J^{i}$, we have that $J^{i} \leq S$. Moreover, we can find a nonsingular submatrix of dimension $J^{i} \times J^{i}$. Specifically, we may assume, without loss of generality, that this matrix is given by

$$
B^{i}=\left|\begin{array}{ccc}
R_{1,1} & \cdots & R_{1, J^{i}} \\
\vdots & \vdots & \vdots \\
R_{J^{i}, 1} & \cdots & R_{J^{i}, J^{i}}
\end{array}\right|
$$

Thus, we have that

$$
\left[\begin{array}{c}
p_{1}\left(x_{1}^{i}-w_{1}^{i}\right) \\
\vdots \\
p_{J}\left(x_{J^{i}}^{i}-w_{J^{i}}^{i}\right)
\end{array}\right]=B\left[\begin{array}{c}
\kappa_{1} \\
\vdots \\
\kappa_{J^{i}}^{i}
\end{array}\right]
$$


By Cramer's Rule,

$$
\kappa_{j}^{i}=\frac{\operatorname{det}\left(B^{i}\left(y^{i}, j\right)\right)}{\operatorname{det}\left(B^{i}\right)}, \quad \forall j \in\left\{1, \ldots, J^{i}\right\},
$$

where $y^{i}=\left(p_{1}\left(x_{1}^{i}-w_{1}^{i}\right), \ldots, p_{J}\left(x_{J^{i}}^{i}-w_{J^{i}}^{i}\right)\right)$ and $B^{i}\left(y^{i}, j\right)$ is the matrix obtained by change, in the matrix $B^{i}$, the $j$-ith column for the vector $y^{i}$. Since (i) the determinant is a continuous function, (ii) the vector $y^{i}$ depends continuously on $\left(\left(p_{s}, x_{s}^{i}\right) ; s \in S\right)$, and (iii) vectors $\left(\left(p_{s}, x_{s}^{i}, w_{s}^{i}\right) ; s \in S\right)$ are in a compact space, it follows that vector $\left(k_{j}^{i} ; j \in J^{i}\right)$ is bounded, independently of the value of $\left(\left(p_{s}, x_{s}^{i}, w_{s}^{i}\right) ; s \in S\right)$. Thus, there exists $A>0$, the maximum across the bounds of $\left(k_{j}^{i} ; j \in J^{i}\right)$ for each $i \in I$, which satisfies the conditions of the lemma and depends on $\left(\left(\bar{W}, w_{s}, R_{s, j}\right) ;(s, j) \in\right.$ $S \times J)$.

Following the notation of the previous lemma, let $\bar{\Theta}:=2 \mathrm{~A}$.

The next two lemmas are used to prove that equilibrium asset prices of the generalized game are uniformly bounded. For convenience of notations, let $W_{0}=\left(W_{0, l} ; l \in\right.$ $L$ ) be the vector of aggregated physical resources at $t=0$, where $W_{0, l}:=\sum_{i \in I} w_{0, l}^{i}$.

Lemma 4 Under Assumptions (B) and (C), fix $(\bar{p}, \bar{q}) \in \mathcal{P} \times \mathbb{R}_{+}^{J}$ and suppose that for any agent $i \in I$, there is an optimal solution $\left(\bar{x}^{i}, \bar{\theta}^{i}\right) \in \bar{\Gamma}^{i}$ for his individual problem such that $\bar{x}_{0}^{i} \leq W_{0}$ and $\bar{x}_{s, l}^{i} \leq 2 \bar{W}, \forall(s, l) \in S \times L$. Then, there exists $\bar{Q}>0$, independent of prices, such that $\max _{j \in J} \bar{q}_{j}<\bar{Q}$.

Proof Fix $j \in J$ and let $\mu:=\frac{\min _{(k, l, h) \in S \times L \times I} w_{k, l}^{h}}{2}>0$. Suppose that an agent $i \in I$ for which $j \in J^{i}$ borrows a quantity $\tilde{\theta}_{j}>0$ of asset $j$ such that $R_{s, j} \tilde{\theta}_{j} \leq \mu$, for any $s \in S .{ }^{17}$ This position on asset $j$ reports a quantity of resources which allow agent $i$ to consume at the first period the bundle $w_{0}^{i}+\left(\bar{q}_{j} \tilde{\theta}_{j}\right) \zeta$ and, therefore,

$$
V^{i}\left(\bar{p}, w_{0}^{i}+\left(\bar{q}_{j} \tilde{\theta}_{j}\right) \zeta,\left(0.5 w_{s}^{i} ; s \in S\right)\right) \leq V^{i}\left(\bar{p}, \bar{x}^{i}\right)<V^{i}\left(\bar{p}, W_{0},(2 \bar{W}(1, \ldots, 1))_{s \in S}\right)
$$

On the other hand, Assumption (C) guarantees that there exists $\bar{r}(\bar{p}) \in \mathbb{R}_{+}^{L}$ such that

$$
V^{i}\left(\bar{p}, W_{0},(2 \bar{W}(1, \ldots, 1))_{s \in S}\right)<V^{i}\left(\bar{p}, w_{0}^{i}+\bar{r}(\bar{p}),\left(0.5 w_{s}^{i} ; s \in S\right)\right)
$$

Indeed, following the notation of Assumption (C), the inequality above follows from

$$
\bar{r}(\bar{p})=\bar{r}_{\tilde{\sigma}}\left(\bar{p},\left(W_{0},(2 \bar{W}(1, \ldots, 1))_{s \in S}\right)\right)+W_{0}-w_{0}^{i} \in \mathbb{R}_{+}^{L},
$$

where $\tilde{\sigma} \in(0,1)$ is chosen to satisfy $2 \bar{W} \tilde{\sigma}<\mu$.

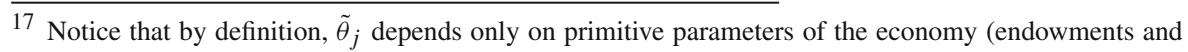
unitary financial payments).
} 
We conclude that

$$
\bar{q}_{j}<Q_{j}(\bar{p}):=\frac{\|\bar{r}(\bar{p})\|_{\Sigma}}{\tilde{\theta}_{j}\|\zeta\|_{\Sigma}} .
$$

Moreover, the upper bound $Q_{j}(p)$ is well defined for any $p \in \mathcal{P}$, and it follows from Assumption (C) that it varies continuously with commodity prices. Thus, the function $Q: \mathcal{P} \rightarrow \mathbb{R}$ defined by $Q(p)=\max _{j \in J} Q_{j}(p)$ is continuous. Since $\mathcal{P}$ is compact, we conclude that there exists $\bar{Q}>0$ such that $\max _{j \in J} \bar{q}_{j}<\bar{Q}$.

We define $\bar{X}=2(1+\bar{Q}) \bar{W}$.

Note that for any $X>\bar{X}$ and $Q>\bar{Q}$, in the associated generalized game $\mathcal{G}(Q, X, \Theta)$ any player $h \in I$ may demand in the first period the bundle used in the proof of Lemma 4. Thus, in this type of generalized game, the existence of an optimal plan satisfying the conditions of lemma above will imply that the unitary prices of assets are bounded from above by $\bar{Q}$.

The existence of equilibria in our economy is a consequence of the following result.

Lemma 5 Under Assumptions (A), (B) and (C), if $(Q, X, \Theta) \gg(\bar{Q}, \bar{X}, \bar{\Theta})$, then every Cournot-Nash equilibrium for $\mathcal{G}(Q, X, \Theta)$ is an equilibrium of the original economy.

Proof Let $\left(\bar{p}, \bar{q},\left(\bar{\eta}^{i} ; i \in I\right)\right)$, where $\bar{\eta}^{i}=\left(\bar{x}^{i}, \bar{\theta}^{i}\right) \in \bar{\Gamma}^{i}$, be a equilibrium for the generalized game $\mathcal{G}(Q, X, \Theta)$, with $(Q, X, \Theta) \gg(\bar{Q}, \bar{X}, \bar{\Theta})$.

Step I: Market feasibility. Aggregating agent's first period budget constraints, we have

$$
\bar{p}_{0} \sum_{i \in I}\left(\bar{x}_{0}^{i}-w_{0}^{i}\right)+\sum_{i \in I} \sum_{j \in J^{i}} \bar{q}_{j} \bar{\theta}_{j}^{i} \leq 0 .
$$

It follows that if $\sum_{i \in I}\left(\bar{x}_{0, l}^{i}-w_{0, l}^{i}\right)>0$, then the auctioneer $h(0)$ will choose the greater price for this good, $\bar{p}_{l}=1$, and zero prices for the other goods and assets, making his objective function positive, which contradicts the inequality above. Therefore, $\sum_{i \in I} \bar{x}_{0}^{i} \leq \sum_{i \in I} w_{0}^{i}<W_{0}$. Analogously, if $\sum_{i \in I(j)} \bar{\theta}_{j}^{i}>0$, then the auctioneer $h(0)$ would choose the maximum price possible for this asset, i.e., $\bar{q}_{j}=$ $Q>\bar{Q}$, which is a contradiction with the result of Lemma 4. Thus, for any $j \in J$, $\sum_{i \in I(j)} \bar{\theta}_{j}^{i} \leq 0$.

Since first period consumption is bounded from above by the aggregate endowment, which is less than $X$, it follows that budget constraints at $t=0$ are satisfied with equality. Hence, the auctioneer $h(0)$ has an optimal value equal to zero. As a consequence, if $\sum_{i \in I}\left(\bar{x}_{0, l}^{i}-w_{0, l}^{i}\right)<0$, the auctioneer $h(0)$ would choose a zero price for the good $l$, a contradiction with the strict monotonicity of preferences for some $i \in I$ (Assumption B). Therefore, $\sum_{i \in I} \bar{x}_{0}^{i}=W_{0}$. Furthermore, if $\sum_{i \in I(j)} \bar{\theta}_{j}^{i}<0$, the auctioneer would choose $\bar{q}_{j}=0$, a contradiction with the monotonicity of preferences. Then, market feasibility conditions hold at $t=0$ in both physical and financial markets. 
Using the market feasibility of $\left(\left(\bar{x}^{i}, \bar{\theta}^{i}\right) ; i \in I\right)$ at $t=0$, and aggregating budget constraints at $s \in S$, we obtain that $\bar{p}_{s} \sum_{i \in I}\left(\bar{x}_{s}^{i}-w_{s}^{i}\right) \leq 0$. Therefore, analogous arguments to those made above ensure that $\sum_{i \in I}\left(\bar{x}_{s}^{i}-w_{s}^{i}\right) \leq 0$. This last property guarantees that budget constraints are satisfied as an equality in the state of nature $s$. Finally, if $\sum_{i \in I}\left(\bar{x}_{s, l}^{i}-w_{s, l}^{i}\right)<0$, then the auctioneer $h(s)$ would choose a zero price for the good $l \in L$, which contradicts individual optimality under strictly monotonic preferences for some $i \in I$. We conclude that market feasibility also holds at each state of nature $s \in S$.

Step II. Optimality of individual allocations. Since market feasibility holds in physical markets, it follows that $\bar{x}_{0, l}^{i}<X$ and $\bar{x}_{s, l}^{i}<2 \bar{W}$, for any $(i, s, l) \in I \times S \times L$. Using Lemma 3, we have that for any $i \in I$ and $j \in J^{i},\left|\theta_{j}^{i}\right|<\Theta$. Thus, for any $i \in I, \bar{\eta}^{i}$ belongs to the interior of $K^{i}(X, \Theta)$.

Suppose that there exists another allocation $\eta^{i} \in \mathbb{R}_{+}^{L \times S^{*}} \times \mathbb{R}^{J^{i}}$ such that $V^{i}\left(\bar{p}, \eta^{i}\right)>$ $V^{i}\left(\bar{p}, \bar{\eta}^{i}\right)$. Since for $\lambda \in(0,1)$ sufficiently small, $\eta^{i}(\lambda):=\lambda \eta^{i}+(1-\lambda) \bar{\eta}^{i} \in$ $K^{i}(X, \Theta)$, the strictly concavity of $V^{i}(\bar{p}, \cdot)$ implies that $V^{i}\left(\bar{p}, \eta^{i}(\lambda)\right)>V^{i}\left(\bar{p}, \bar{\eta}^{i}\right)$, a contradiction with the optimality of $\bar{\eta}^{i} \in \bar{\Gamma}^{i}$. Therefore, for any $\eta^{i} \in \mathbb{R}_{+}^{L \times S^{*}} \times \mathbb{R}^{J^{i}}$, $V^{i}\left(\bar{p}, \eta^{i}\right) \leq V^{i}\left(\bar{p}, \bar{\eta}^{i}\right)$, which proves the optimality of $\bar{\eta}^{i} \in B^{i}(\bar{p}, \bar{q})$ among the allocations in the agent $i$ 's budget set. Notice that as was proved in Sect. 3, informational compatibility of consumption allocations follows from Assumption (A).

\section{References}

Aouani, Z., Cornet, B.: Existence of financial equilibria with restricted participation. J. Math. Econ. 45, 772-786 (2009)

Aouani, Z., Cornet, B.: Reduced equivalent form of a financial structure. J. Math. Econ. 47, 318-327 (2011)

Cass, D.: Competitive equilibrium with incomplete financial markets. J. Math. Econ. 42, 384-405 (2006). See also 1984 CARESS working paper with identical title

Condie, S., Ganguli, J.: Ambiguity and rational expectations equilibria. Rev. Econ. Stud. 78, 821-845 (2011)

Cornet, B., Gopalan, R.: Arbitrage and equilibrium with portfolio constraints. Econ. Theor. 45, 227-252 (2010)

Cornet, B., Ranjan, A.: Existence of financial quasi-equilibria with restricted participation. In: Working Paper, Paris School of Economics (2011)

Correia-da-Silva, J.: Two-period economies with price-contingent deliveries. Econ. Theor. 59(3), 509-525 (2015)

Correia-da-Silva, J., Hervés-Beloso, C.: Subjective expectations equilibrium in economies with uncertain delivery. J. Math. Econ. 44(7-8), 641-650 (2008)

Correia-da-Silva, J., Hervés-Beloso, C.: Prudent expectations equilibrium in economies with uncertain delivery. Econ. Theor. 39(1), 67-92 (2009)

Correia-da-Silva, J., Hervés-Beloso, C.: Irrelevance of private information in two-period economies with more goods than states of nature. Econ. Theor. 55(2), 439-455 (2014)

De Castro, L., Pesce, M., Yannelis, N.: A new perspective on rational expectations. In: Working Paper (2014)

Dubey, P., Geanakoplos, J., Shubik, M.: The revelation of information in strategic market games. J. Math. Econ. 16, 105-137 (1987)

Einy, E., Moreno, D., Shitovitz, B.: Rational expectations equilibria and the ex-post core of an economy with asymmetric information. J. Math. Econ. 34, 527-535 (2000)

Einy, E., Moreno, D., Shitovitz, B.: Competitive and core allocations in large economies with differential information. Econ. Theor. 18, 321-332 (2001) 
Faias, M., Moreno-García, E.: Incomplete financial markets and differential information. Econ. Theor. 43, 189-206 (2010)

Pollak, R.: Price dependent preferences. Am. Econ. Rev. 67, 64-75 (1977)

Radner, R.: Équilibre des marchés a terme et au compatant en cas d' incertitude. Cahiers du Séminaire d' Économétrie 9, 35-52 (1967)

Radner, R.: Competitive equilibrium under uncertainty. Econometrica 36, 31-58 (1968)

Radner, R.: Rational expectations equilibrium: generic existence and the information revealed by prices. Econometrica 47(3), 655-678 (1979)

Seghir, A., Torres-Martínez, J.P.: On equilibrium existence with endogenous restricted financial participation. J. Math. Econ. 47, 37-42 (2011) 